\title{
Groundwater flow on a drained beach
}

\author{
A. Saponieri \& L. Damiani \\ Dipartimento di Ingegneria Civile, Ambientale, del Territorio, Edile e di \\ Chimica (DICATECh), Politecnico di Bari, Italy
}

\begin{abstract}
This paper deals with the analysis of the groundwater flow on a drained beach. Infiltration processes in static conditions (without running waves) are numerically simulated by using HYDRUS-2D software. Nevertheless, field and laboratory experiments show the possibility of studying the influence of the system on both nearshore hydrodynamics and morphodynamics using a numerical model able to reproduce the drainage main physical processes. It is fundamental in order to define the main Beach Drainage System (BDS) design criteria which, to date, are not yet defined. The groundwater flow through a partially saturated porous media is described using Richard's equation. Numerical results are then compared with the experimental ones obtained during an intensive experimental program performed on a BDS prototype at the GrosserWellen Kanal (GWK) in Hannover. In particular, the study focuses on the BDS influence on the groundwater table inside the beach and drained flows.
\end{abstract}

\section{Introduction}

Coastal erosion is the result of a complex interaction of natural processes, furthered by human actions. In particular, the cross-shore sediment transport plays a dominant role in the morphological evolution of sandy beach profiles, above all in the nearshore zone. In general, the beach morphological evolution is strongly influenced by several parameters, such as wave conditions, tide levels, bathymetry, sand grain size, initial beach profile and sediment distribution along the profile.

The study of sediment transport across the swash zone is difficult, especially when the influence of simultaneous physical processes (such as swash flow velocity, water depth variation, infiltration and groundwater flow) has to be considered. Grant [1] first suggested a link between the beach groundwater 
behavior and the swash zone sediment transport. A critical factor of swashgroundwater interaction is the elevation of the beach groundwater table relative to the mean sea level. A relative low water table is thought to enhance the swash infiltration and, as a consequence, the onshore sediment transport. On the contrary, a high water table suggests a promotion of the swash ex-filtration and the offshore sediment transport.

When a beach experiences long-period waves, the run-up flow can infiltrate into the porous medium, causing an increase of the effective stresses and, thus, of the sediment stability, by favoring the settlement of the suspended load. On the contrary, when the beach experiences winter storms, it quickly reaches its maximum saturation degree. In such a condition, the backwash flux prevails over the up-rush one because the water can not infiltrate through the sand. The net result of the run-up/down processes is an offshore sediment transport causing, consequently, the shore erosion.

The Beach Drainage System (BDS) is born as an auxiliary system for the management of the soft solution (i.e. nourishment) against beach erosion.

In general the system aims to interact with the swash hydrodynamics by favoring the deposition of sediments transported by waves during the up-rush phase and contrasting their offshore movement during the backwash. It artificially increases the sand permeability, producing a groundwater table lowering and an increase in the thickness of the unsaturated zone. Moreover, the vertical infiltration of the sea water, also allows the effective stresses carried out by the soil skeleton to be increased, enhancing the beach stability.

Several field installations of BDS are available at many sites around the world. The analysis of systems response shows that the effective beach stabilization is still not well defined. Although the sites have different wave climates and morphological features, the BDS have almost the same design parameters with different responses in system efficacy [2]. The lack of definitive BDS design guidelines is mainly due to a discontinuous long-term monitoring and to few laboratory experiments available in the literature. To date, all design parameters (number of pipes, pipe diameter, excavation depth and location, etc.) are only based on sellers technician acquired experiences.

For this purpose, laboratory models are necessary, paying particular attention on model scale, especially for parameters like grain size and hydraulic conductivity, which, typically in movable-bed models, have a great influence in sand mechanical behavior understanding.

A set of laboratory tests on a 1:1 BDS model was performed at the Grosser WellenKanal (GWK) of Hannover University. The projects involved a series of experiments aimed at testing drain systems under different wave conditions.

A mathematical study of the infiltration processes inside a partially saturated porous medium represents the first step for developing a model able to provide the main Beach Dewatering System design guidelines. For this reason, laboratory experiments have to be combined with numerical modeling in order to extrapolate information from a limited number of field and laboratory experiments to different drain configurations, wave climates and beach characteristics. 


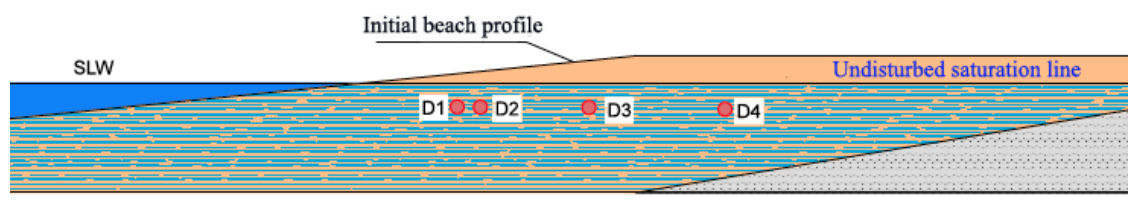

Figure 1: Sketch of a part of the BDS physical model at GWK.

The present work deals with a numerical simulation of the groundwater flow in a drained beach. Then a numerical model is discussed and first results on comparison between measured and simulated groundwater flow are shown. In particular, attention is focused on both saturation line behavior and flows in drained conditions.

\section{The physical model}

The laboratory investigation of a Beach Drainage System full-scale physical model was performed at the Large Wave Flume (Grosser WellenKanal, GWK) in Hannover. The model was designed on the basis of the previous experiments performed in Bari [3]. A complete and detailed description of experiments, measurements methods and results is explained in other works $[2,4,5])$.

Figure 1 shows the physical model close to the drainage system. Grain size curve and main sand characteristics were analyzed at the laboratories of Politecnico of Bari. The median diameter $D_{50}$, the fall velocity and the permeability are equal to $0.33 \mathrm{~mm}, 4.8 \mathrm{~cm} / \mathrm{s}$ and $3.2 * 10^{-2} \mathrm{~cm} / \mathrm{s}$, respectively. The measure of sand characteristic creates some doubts because of the hight distortion during the sand sampling. For this reason, preliminary numerical tests have been performed with different values of permeability, confirming the great influence of the permeability on results. The adopted drainage system was constituted by four corrugated polyvinyl chloride (PVC) drains placed inside the beach, under the static water level, in correspondence to the swash zone and transversally to the incident wave motion (parallel to the shoreline). Moreover, drains were covered by a double layer of geotextile in order to avoid blockage due to the sand during water drainage which could put the system out of action. Drained water was then transported to a pumping well and discharged into the flume by an external iron pipe. Two sets of tests were performed. The first one with two different undisturbed water levels in the channel equal to $4.00 \mathrm{~m}$ (Test S1) and $4.20 \mathrm{~m}$ (Test S2). Tests S2 were performed in order to simulate the wave set-up due to the sea-tide. During the second set of tests the drainage system was tested under different wave energy conditions.

Both morphodynamic and hydrodynamic behaviors of a drained beach were analyzed by using the data sampled by a large number of instruments. Surface elevation, pressure, velocity, drainage and beach profile were measured in order to delve into the nearshore processes. In particular, water table oscillations were 
recorded using both piezometers and pore pressure transducers, placed inside the sand along the beach profile at the same drain depth. Drained flows were calculated by means of a flowmeter placed on the external pipe and a pore pressure transducer placed into the pumping well.

\section{The mathematical model: the HYDRUS-2D software}

In the present study the mathematical model focuses on the groundwater flow evaluation by using Richard's equation numerically solved by the code HYDRUS$2 D$.

The HYDRUS-2D software package simulates two-dimensional water flow, heat movement and transport of solutes involved in sequential first-order decay reactions in unsaturated, partially saturated or fully saturated porous media. The program can handle flow domains delineated by irregular boundaries and the flow region may be composed of non-uniform soils having an arbitrary degree of local anisotropy. Saturated-unsaturated water flow and convection-dispersion type equations for heat and solute transport are described by Richard's equation, solved by the Galerkin-type linear finite element schemes. The code also includes a Marquardt-Levenberg parameter optimization algorithm for inverse estimation of soil hydraulic and solute transport and reaction parameters from measured transient or steady-state flow.

Considering two-dimensional isothermal Darcian water flow in a partially saturated rigid porous medium and assuming that the air phase can be neglected in the liquid flow process, the governing equation [6] is given by the following modified form of Richard's equation (1):

$$
\frac{\partial \vartheta}{\partial t}=\frac{\partial}{\partial x_{i}}\left[K\left(K_{i j}^{A} \frac{\partial h}{\partial x_{j}}+K_{i z}^{A}\right)\right]-S
$$

where $\vartheta$ is the volumetric water content $\left[L^{3} L^{-3}\right] ; t$ is the time $[T] ; h$ is the pressure head $[L] ; K_{i j}^{A}$ are the components of a dimensionless anisotropy tensor $K^{A}$ in which the diagonal of entries is equal to one and the off-diagonal entries equal to zero for an isotropic medium; $x_{i}(i=1,2)$ are the spatial coordinates $[L]$; $S$ is a sink term $\left[T^{-1}\right]$ and it represents the water volume removed per unit time from a unit soil volume due to plant water uptake; $K$ is the unsaturated hydraulic conductivity function $\left[L T^{-1}\right]$. The solution of Equation (1) requires knowledge of the initial distribution of the pressure head within the flow domain. HYDRUS-2D implements three types of conditions to describe system-independent interactions along the flow region boundaries (pressure head, flux and gradient boundary conditions). In addition, there is the possibility of imposing other three different types of system-dependent boundary conditions. The solution of the governing flow equation (1) is obtained by using the Galerkin finite element method with linear basis function [7].

In the present work only the static conditions have been considered in order to validate the model by using the experimental data collected during GWK experiments. 


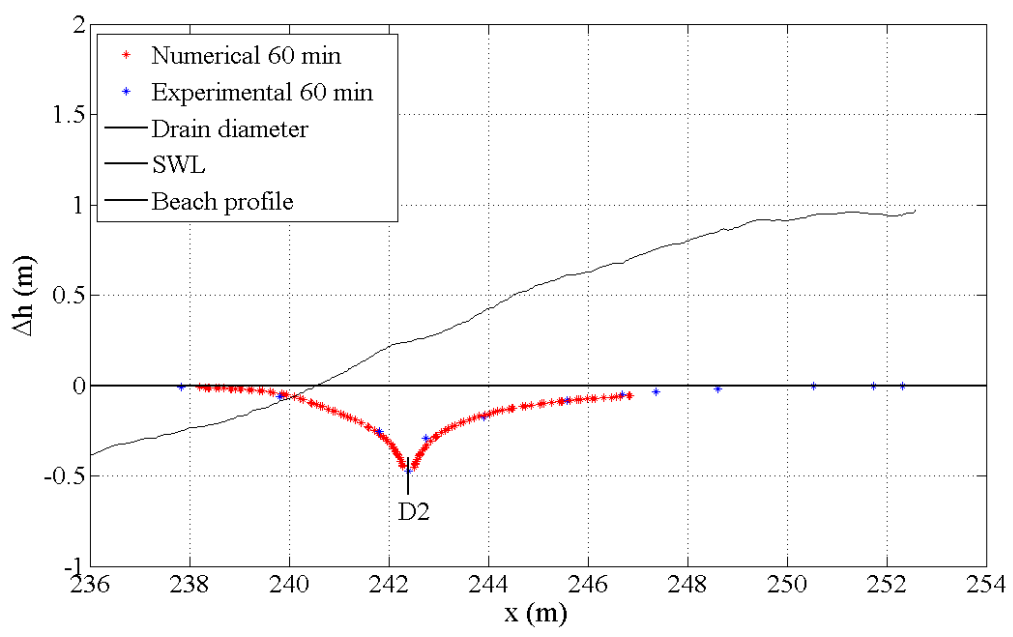

Figure 2: Spatial variation of saturation line - Drain D2 Test S1.

The geometry reproduces the physical model adopted for GWK tests. The finiteelement mesh is constructed by dividing the beach profile into triangular elements. The mesh is almost uniform except in the zone close to the drain where a local refinement has been used in order to obtain more accurate results.

The beach is assumed homogeneous and isotropic. Running the model requires the initial water content distribution as well as the soil hydraulic parameters. Standard values depending on the soil type are used. Only the hydraulic conductivity has been imposed.

The initial water content distribution has not been set a priori. In order to reproduce the real laboratory initial conditions, the channel filling-up has been simulated. For such simulations constant hydraulic heads (equal to $4.00 \mathrm{~m}$ and $4.20 \mathrm{~m}$, respectively) have been imposed on the beach bottom, causing the gradual beach saturation from the bottom to the top. The output of this simulation represents the initial hydraulic head distribution in the porous medium for drainage simulation.

The drains are modeled as circular holes in the mesh. All beach contours are no flux boundaries, while each drain is considered as a free drainage surface. The code assumes that as long as a drain is located in the saturated zone, the pressure head along the drain is equal to zero. On the left side the static water levels have been imposed constant during the drainage processes. The total time of each simulation has been set at $60^{\prime}$ (as for laboratory runs) and the time discretization $\Delta t$ has be set equal to 0.001. A smaller time interval $\Delta t$ cause the bad convergence of the numerical solution. 


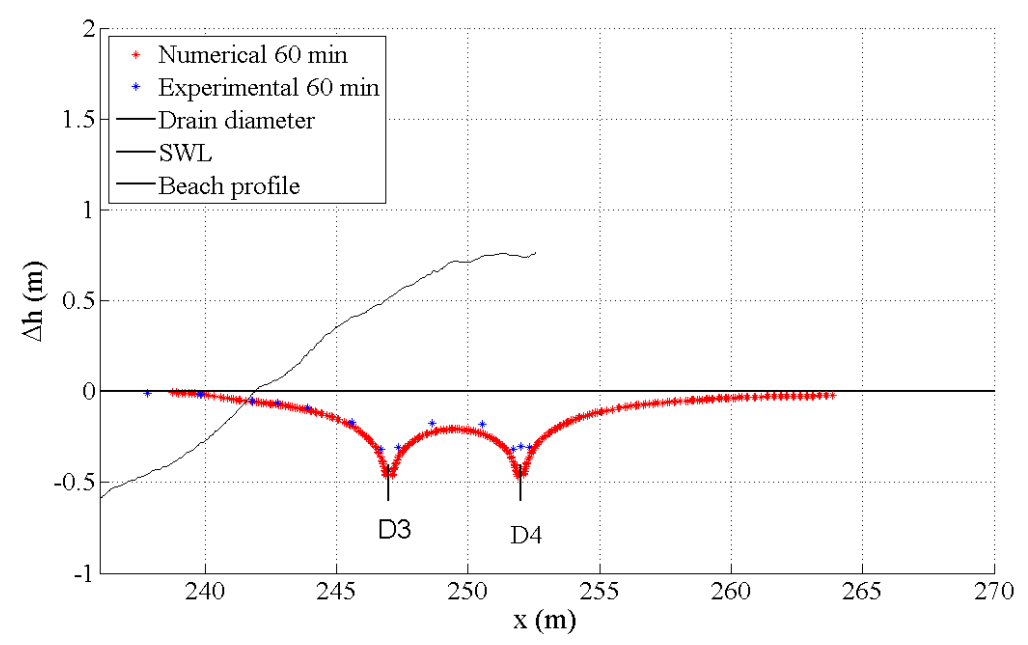

Figure 3: Spatial variation of saturation line - Drains D3+D4 Test S1.

\section{Results}

Figure 2 reports both numerical and experimental groundwater table lowering $(\Delta h)$ with respect to the initial static level $S 1=4.00 \mathrm{~m}$ due to the single working drain D2 at the end of the run $\left(t=60^{\prime}\right)$. Moreover, the beach profile measured in the laboratory at the end of the test is reported. Red points represent the spatial variation of the water table modeled by the numerical code, while blue stars represent the local piezometric head variation measured in the laboratory. It is evident that in both cases the drain effect on the groundwater behavior is the saturation line lowering, resulting in an increasing of the unsaturated area inside the beach. The drain influence decreases moving away from the section in which the drain is placed. It tends to the Static Water Level (SWL) seawards and to the undisturbed water table in the inner part of the beach.

The comparison between the numerical results and the experimental data shows that the numerical model well simulates the experimental water table lowering due to the drainage. In general, laboratory measurements show a slightly smaller water table lowering. The difference is negligible with respect the total hydraulic head loss, especially in the zone close to the drain. This behavior can be observed in all single drains configurations.

As previously mentioned, the system was tested in different drain configurations, also with simultaneous working drains. As an example, Figure 3 reports both numerical and measured water table spatial variation for D3 and D4 working all at once. The two drains opened simultaneously were tested in order to evaluate the possible advantages in using more drains in field applications. The addition of D3 produces an increase of the unsaturated area. In this case the distance between the two drains leads to an increase of the water table between 


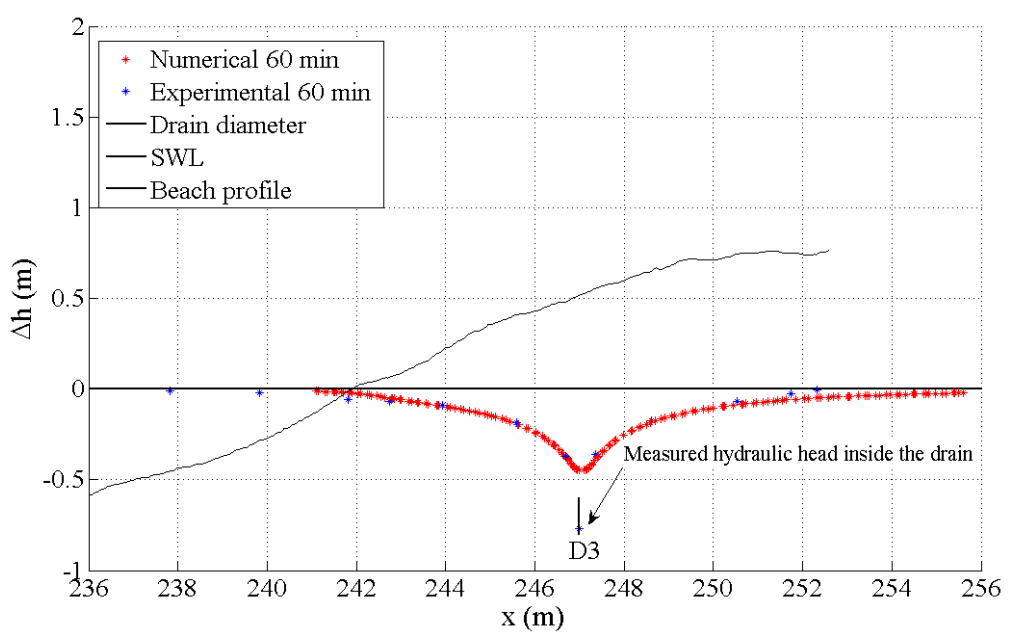

Figure 4: Spatial variation of saturation line - Drain D3 Test S2.

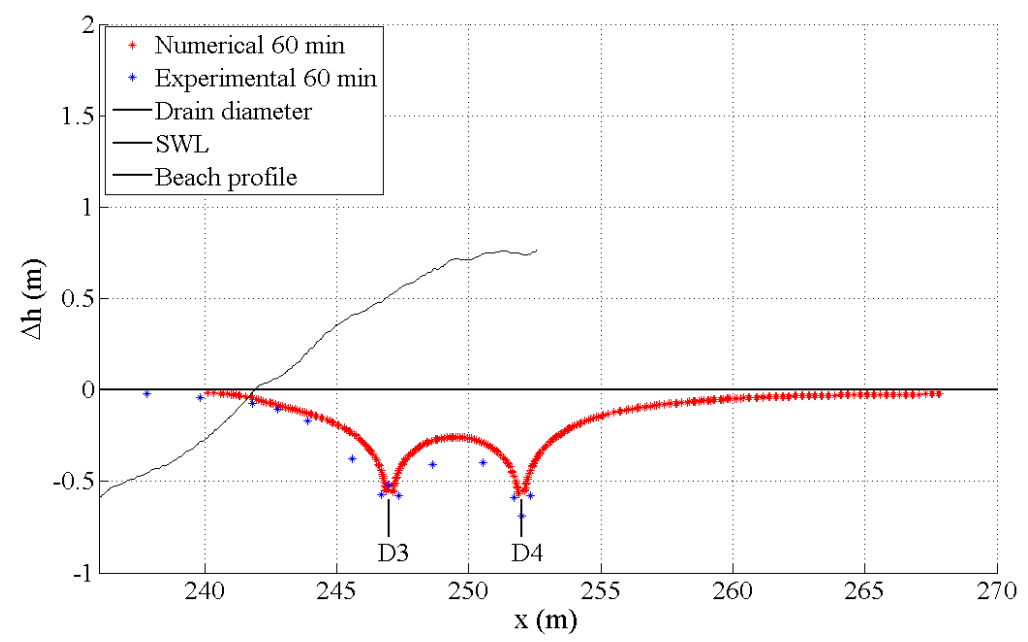

Figure 5: Spatial variation of saturation line - Drains D3+D4 Test S2.

the two drains, which is more relevant in laboratory tests. The above system configurations were also tested with a higher static level equal to $4.20 \mathrm{~m}$ (Test S2). Figure 4 reports the water table spatial variation with working drain D3. As well as tests $\mathrm{S} 1$, when the SWL in the channel is higher, numerical and laboratory data show a good agreement. During tests S1, drains are able to desaturate the entire sand column above them. With the increasing of the SWL the drains are not able to desaturate the entire water column above them. The saturation line, indeed, does not reach pipe contours in all drain conditions. 


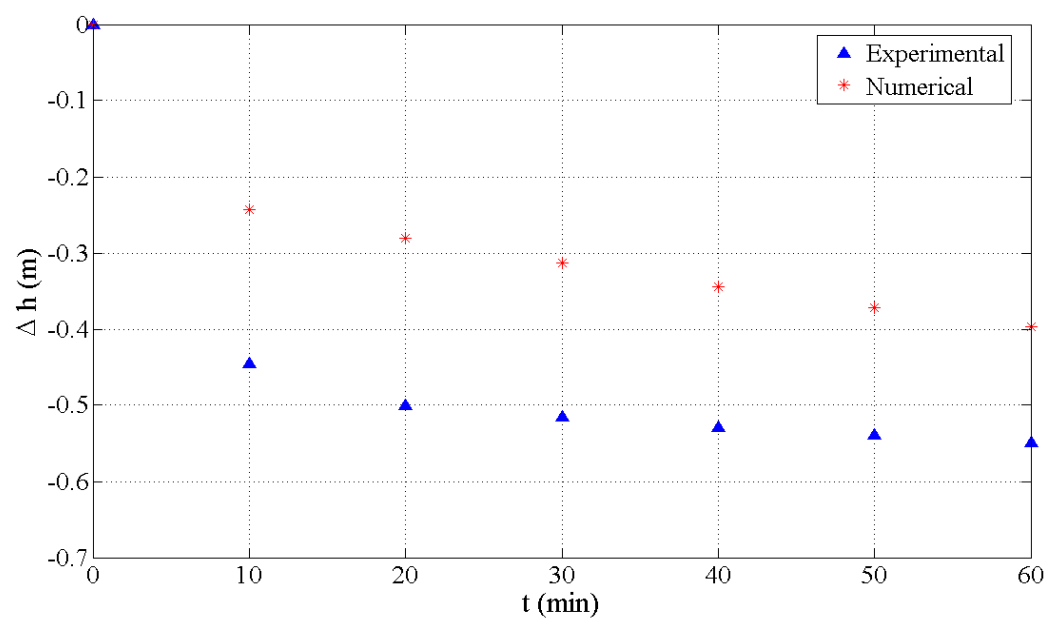

(a) Time variation

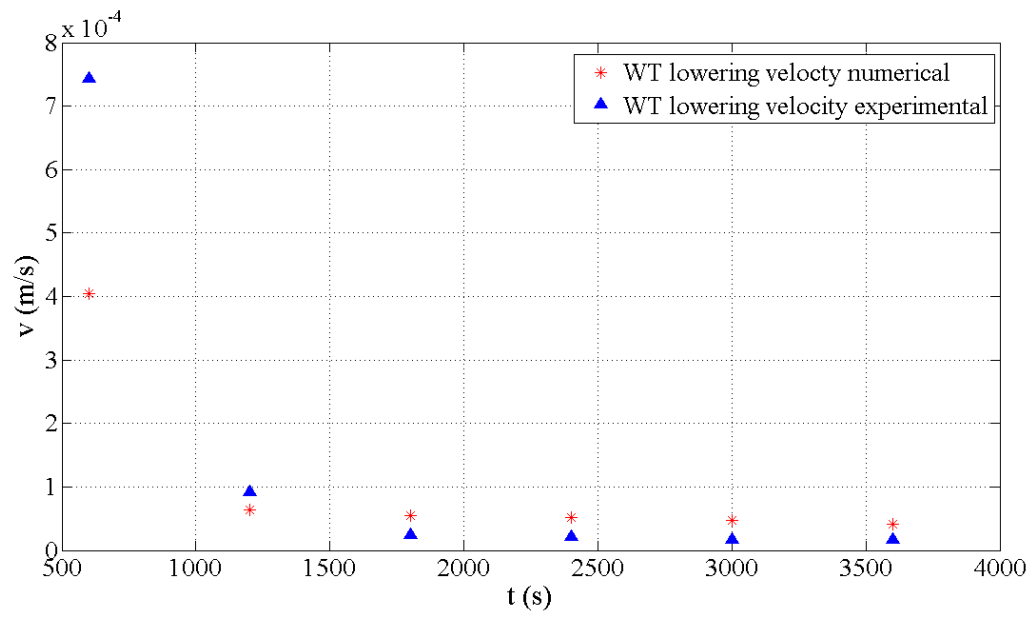

(b) Water table lowering velocity

Figure 6: Water table lowering D3 on - test S2.

Figure 4 also shows the hydraulic head measured in the laboratory by a pore pressure transducer placed inside the drain. Up to now the flow regime inside the drains has not yet been deeply analyzed. Previous experimental [3] and numerical [8] BDS modeling aimed to analyze only the dynamic fluctuations inside the beach, but did not clarify the real drain flow regime, assuming the same hydraulic heads outside and inside. The pressure transducers placed inside the drains during full scale experiments at GWK allow for measurement of the hydraulic head 


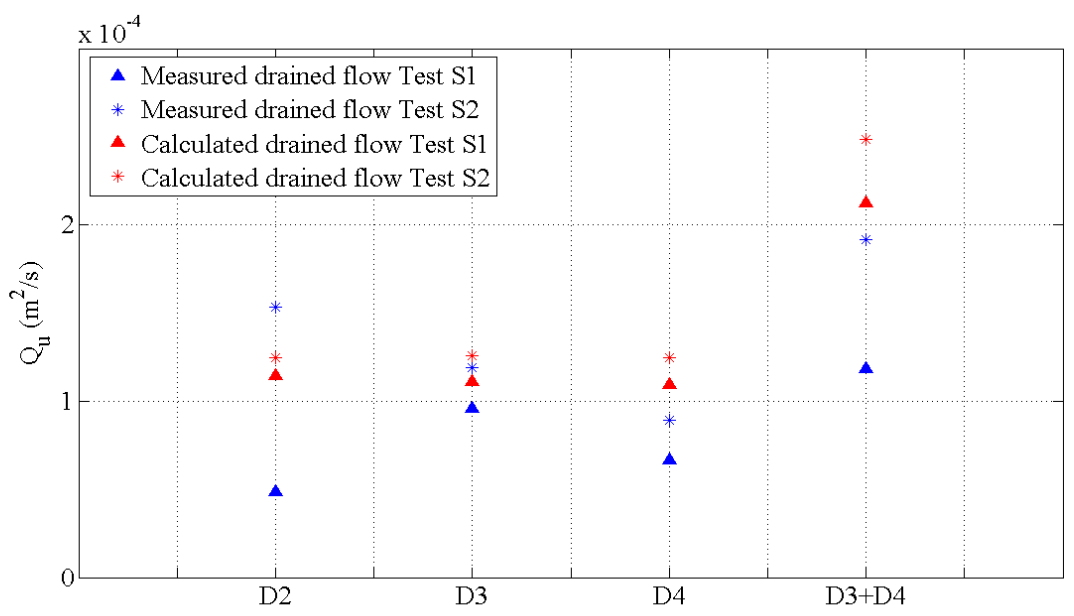

Figure 7: Calculated and measured drained flows.

inside. In all tested drain configurations with a SWL equal to $4.20 \mathrm{~m}$ obtained results indicate that the hydraulic regime inside the drains proves to be probably different from that one developed in the sand. This behavior might be related to the energy losses induced by the water during infiltration processes, probably mainly due to pipe characteristics and covering system (geotextile). The system behavior with more drains working simultaneously was tested also with the higher static water level. Figure 5 reports the spatial variation of water table at the end $\left(t=60^{\prime}\right)$ of static test S2 with D3 and D4 opened. The addition of drain D4 to D3 causes a small increase of water table lowering. Also with a higher water level in the channel, the most noticeable effect of using more drains together is a major reduction of the saturated area.

Figure 6 shows an example of time variation of water table lowering (Figure 6(a)) and the corresponding lowering velocity (Figure 6(b)) numerically calculated (red stars) and measured in the laboratory (blue triangles) in correspondence of the piezometer placed near the drain D3, for the test S2. Both numerical and experimental data show that the water table decreases quicker in the first 10 minutes, then reaching a quasi-steady condition. In all tested drain configurations the $70 \div 80 \%$ of water table lowering occurs in the first 10 minutes.

Figure 7 reports both experimental (blue stars and triangles) and numerical (red stars and triangles) drained flows. The drained discharges are higher for S2 with respect to S1 in both cases. The higher drained flows measured for static tests S2 correspond to a deeper water table lowering, when drains work singularly. When D3 and D4 work simultaneously, higher values of drained flows are not only a function of the maximum water table but they mainly depend on the whole unsaturated area above the drains. Experimental data have a poorly-defined trend during the activation of each drain. It is not possible, indeed, to define a relationship between the drained flows and the pipe distance from the initial 
shoreline position. On the contrary, in numerical simulation, the drained flow seems to be not influenced by the drain position. Drained flows assume about the same value.

\section{Conclusions}

The present work deals with the study of groundwater flow in a beach equipped with a Beach Drainage System (BDS). Infiltration processes in a drained beach are numerically simulated by means of the HYDRUS-2D code. Obtained data are compared with experimental data collected during laboratory tests performed at the laboratory GWK of Hannover University. The present study focuses on water table and drained flows analysis in static conditions. The numerical model simulates quite well the water table lowering. Drained flows are also almost comparable. Measured drained flows show a more evident influence of drain distance on drainage with respect to the numerical results. Moreover, the study underlines the influence of drain characteristics on the system efficacy.

The present study represents the first step for a complete numerical modeling of the phenomena involved in a drainage process. The analysis of static conditions is mainly aimed at correlating the system characteristics (drain diameter, alitmetric and planimetric position, geotextile, etc) to the system capacity in draining water. Future applications will regard the possibility of improving the adopted numerical model by simulating the drainage efficiency under different wave attacks, in order to correlate the drainage efficiency to the wave-climate conditions and to define the main BDS guidelines.

\section{References}

[1] Grant, U.S., Effects of groundwater table on beach erosion, Bulletin of the Geological Society of America, 57, 1946.

[2] Saponieri, A., Hydrodynamic and Morhodynamic study of a drained beach, PhD Thesis, 2012.

[3] Damiani, L., Petrillo, A.F. and Saponieri, A., Beach dewatering systems:Modelling coastal groundwater flow, Proceedings of 33rd IAHR Congress: Water Engineering for a sustainable environment, pages 7304 7311, Vancouver, British Columbia, August 2009.

[4] Damiani, L., Aristodemo, F., Saponieri, A., Verbeni, B., Veltri, P., and Vicinanza, D., Full-scale experiments on a beach drainage system: hydrodynamic effects inside beach, Journal of Hydraulic Research, 49(1):4454, 2011.

[5] Damiani, L., Vicinanza, D., Aristodemo, F., Saponieri, A., and Corvaro, S., Experimental investigation on wave set-up and nearshore velocity field in presence of a BDS, Journal of Coastal Research, SI 64:55-59, 2011. 
[6] Simunek, J.,Senja, M. and van Genuchten, Th., HYDRUS-2D Simulating water flow, heat and solute transport in two-dimensional variably saturated media., Colorado School of Mines, 1999.

[7] Neuman, S.P., Galerkin approach to saturated-unsaturated flow in porousmedia., Chapter 10 in Finite elements in fluid, Volume 1. Wiley, 1975.

[8] Karambas, T.V., Numerical study of the beach profile evolution due to a coastal drain system., In Proceedings of 33rd IAHR Congress: Water Engineering for a sustainable environment, pages 1-8, Vancouver, British Columbia, August 2009. 